\title{
La solitude en partage. Éthique et communauté chez Pascale Kramer
}

\author{
Anne-Frédérique SCHLÄPFER \\ Fonds national suisse
}

\begin{abstract}
Depuis ses débuts, Pascale Kramer explore les liens entre individus et collectifs, entre le micro et le macrosocial. La question de la communauté occupe dès lors une place centrale dans ses romans et s'adosse à une réflexion sur l'usage du pronom " nous ». En mettant en scène des personnages marginaux, dont le lecteur est invité à épouser le point de vue, l'auteure nous incite d'une part à éprouver leurs souffrances et leur solitude et, de l'autre, à imaginer des manières de réactiver les liens de solidarité dans le monde réel, conférant ainsi une véritable puissance à la littérature. En cela, Kramer s'inscrit dans le tournant critique du roman français contemporain et rejoint les préoccupations éthiques de philosophes tels que Richard Rorty.
\end{abstract}

Keywords : Pascale Kramer, communauté, éthique, «nous », imagination, roman contemporain

La question de la communauté, qu'elle soit abordée par le prisme des liens de solidarités ou à travers des réflexions portant davantage sur les implications éthiques et politiques des sciences humaines et de la littérature irrigue depuis plus d'une vingtaine d'années les champs de la philosophie et des études littéraires. On doit à des auteurs anglo-saxons ayant opéré ce qu'on a nommé le "tournant éthique »-Richard Rorty, Martha Nussbaum, Cora Diamond ou Wayne C. Booth - d'avoir initié ce que le premier décrit comme un « retournement général de la théorie au profit de la narration » (Rorty 1993: 17). Dénonçant une scientificité des discours qu'il estime factice et les apories des grandes théories morales, Rorty se propose d'explorer les bénéfices qu'offre la fiction pour la création de nouvelles solidarités humaines qu'il souhaite plus démocratiques et plus justes. Ce projet, que le philosophe reconnaît tenir en partie de l'utopie, est à l'origine d'une démarche à la fois pragmatique, éthique et politique. Il s'agit, par la fréquentation des arts, mais surtout, en ce qui nous concerne ici, de la littérature, d'élargir « le cercle de nos connaissances» (Rorty 1993: 120) afin de connaître et d'éprouver des situations avec lesquelles nous ne sommes pas familiers et de renforcer, ainsi, la cohésion sociale. L'imagination littéraire, selon lui, sensibilise et favorise donc la compréhension d'autrui, moins sous la forme d'une identification totale que d'un rapprochement momentané :

Pour en venir à voir d'autres êtres humains comme "des nôtres ", plutôt que des « eux », il faut une description minutieuse de ce à quoi ressemblent ces 
êtres qui nous sont peu familiers et une redescription de ce à quoi nousmêmes nous ressemblons. C'est une tâche qui relève, non pas de la théorie, mais de genres tels que l'ethnographie [...] et surtout le roman(Rorty I993: I7).

$\mathrm{Du}$ fait que les genres narratifs proposent un réalisme qui se traduit dans la richesse des détails et dans la finesse des descriptions, ils invitent les lecteurs à prendre en considération l'existence et les souffrances de ceux qu'ils ignorent dans la réalité. Émergent alors des communautés temporaires, imaginées et affectives, ou des «nostrations » dans les termes de Jean-Christophe Bailly (20I4 : I76). Ces communautés dont les bords restent flous, garantiraient toutefois une solidarité susceptible de modifier, en retour, les rapports interpersonnels. Comme Rorty, Bailly envisage la création « d'un nous réel et sans durée, d'un nous qui ne s'installe pas, qui ne se replie pas sur lui-même » (20I4 : I77). Néanmoins, ces communautés provisoires n'existent qu'à la condition que le lecteur soit capable d'imagination, de compassion et d'empathie, car, comme le note encore Rorty :

[l]a solidarité ne se découvre pas par la réflexion, elle se crée. Elle se crée en devenant plus sensible aux détails particuliers de la douleur et de l'humiliation d'autres types de personnes [...]. Cette sensibilité accrue aidant, il devient plus difficile de marginaliser des personnes différentes de nous (1993: I7).

C'est à cette double question de l'imagination et de l'empathie que Nussbaum a consacré plusieurs ouvrages dans lesquels elle montre ce que l'on gagne à penser que la « littérature modèle l'imagination et les désirs » (Nussbaum 2005 : 28). Si elles divertissent, les œuvres romanesques proposent surtout aux lecteurs que nous sommes de véritables expériences qui, en produisant des « émotions puissantes », "déconcerte[nt] et questionne[nt] » (35). Plus encore, elles influent sur nos choix et nos comportements éthiques quotidiens et font du roman « un genre du progrès » moral (Rorty I993 : I7). Dans ce cadre, la littérature, qui appelle une lecture à la fois empathique et critique, prépare le débat démocratique.

L'imagination, point de jonction entre le privé et le public, entre l'éthique et le politique, la fiction et la réalité, a également suscité l'intérêt des littéraires francophones. Alors que les philosophes anglo-saxons s'appuient volontiers sur des œuvres classiques, principalement issues du XIX ${ }^{\mathrm{e}}$ siècle, qu'ils considèrent comme les vecteurs privilégiés des conflits moraux et des grandes passions ${ }^{\mathrm{I}}$, les spécialistes de la littérature francophone se sont

I Nussbaum note ainsi que le roman réaliste « s'enracine dans l'importance profonde de la vulnérabilité de la vie humaine. [...] sa forme même construit la compassion chez les lecteurs, en fait des personnes qui se préoccupent profondément de la souffrance et du malheur 
emparés de cette question en l'appliquant aux particularités du roman contemporain français, parfois réputé, à tort, sans enjeux critiques². Loin des postures qui régissent les grandes fresques balzaciennes à l'ambition totalisante ou les romans de formation de Dickens, ces fictions proposent un imaginaire qui apparaît comme plus modeste et plus fragmentaire. Elles se concentrent volontiers, comme l'ont noté Bruno Blanckeman (2015) ou Morgane Kieffer (2019), sur la vie de personnages marginaux et donnent à voir, en suivant tour à tour des parcours individuels ou collectifs, les logiques d'exclusion et la précarité grandissante d'une partie de la société. On pourrait également ajouter que de nombreux romanciers tentent de restituer la solitude et les mécanismes d'aliénation qui menacent les relations intimes dans les milieux les plus variés.

Dans ces travaux inspirés du tournant éthique, l'imagination s'articule à la notion d'engagement, pensée hors du cadre établi par Sartre à la fin des années 1940. Il ne s'agit plus de considérer que l'écrivain expose à grands traits les principes de positions ostensiblement militantes et politiques, ni qu'il présente d'invariables passions humaines, mais plutôt qu'il invite ses lecteurs à imaginer et à partager, au travers d'un récit qui fait la part belle à l'ordinaire et au présent, les conditions de groupes évoluant dans les marges de la société et que la littérature représente peu. À en croire Bruno Blanckeman (2015), on passerait ainsi, avec le XXI siècle, d'une esthétique du surplomb, propre à la littérature dite engagée - qui incarne une idéologie clairement identifiable -, à une poétique de l'implication, plus subtile, qui s'exprime en termes de relations et qui renonce à toute forme de jugement définitif. En cela, les romanciers contemporains seraient bien loin du retrait qu'on leur a prêté, eux que l'on a parfois dit friands des facilités de l'autofiction et coupés des problèmes sociaux.

Mais cet investissement éthique de l'auteur ne fonctionne qu'à condition que le lecteur réponde à l'interpellation de la fiction en agissant dans le monde réel ou, à tout le moins, en y portant un regard critique. Et c'est là que de nombreux spécialistes situent l'efficace du roman de ces trente dernières années. Dans un article consacré aux formes du réalisme contemporain, Morgane Kieffer souligne justement combien la portée critique de ce type de romans « ne trouve son aboutissement qu'à la condition d'une lecture

d'autrui, et qui s'identifient à autrui parce que ceux-ci révèlent nos propres possibles ». Et de conclure que « cette évaluation est indispensable à une rationalité sociale complète " (I44-I45). 2 En partant des textes de Virginie Despentes, Patrick Deville, Yannick Haenel, Leslie Kaplan ou encore Christine Montalbetti, des critiques déploient les enjeux critiques du roman contemporain. On pense ici aux articles rassemblés par Catherine Brun et Alain Schaffner dans Des écritures engagées aux écritures impliquées. Littérature française $x x^{e} / x_{x I} I^{e}$ siècles (2015), ainsi qu'aux travaux de Morgane Kieffer, Vincent Message, Dominique Rabaté ou Dominique Viart, entre autres. 
empathique » (20I9: I3), en ce que celle-ci permet au lecteur de réagir aux scènes de la fiction et de s'impliquer en retour (2019: I6).

Le cas de Pascale Kramer s'inscrit pleinement dans cette veine du réalisme romanesque et de ce que Dominique Viart nomme les «fictions critiques » contemporaines (2004 : 29). Dans ses romans, Kramer cherche, en effet, à penser l'appariement de l'individuel et du collectif, de l'éthique et du politique. J'aimerais ainsi montrer comment l'auteure ne cesse d'explorer la fragilité des relations humaines et de la communauté pour inviter le lecteur à en réenchanter les liens dans le monde réel. Elle le fait doublement : en représentant, d'abord, des personnages en prise avec des difficultés sociales et intimes, et pour lesquels la notion même de communauté semble se déliter ; ensuite, en proposant à ses lecteurs de faire l'expérience, par le biais de la fiction, des situations de solitude radicale. Ces deux pans - représentation et expérience - se nouent autour de la question de l'imagination, dont le personnel romanesque semble souvent dépourvu. Les lecteurs doivent alors imaginer à leur tour pour pallier ces carences et créer, dans le monde réel, de nouvelles formes de communauté et, ainsi, permettre de repenser le « nous».

\section{«À quoi se mesur[e] finalement la normalité des vies? »}

Telle est la question que se pose le personnage principal de Gloria (2013), un ancien assistant social. Laissée en suspens, elle est comme adressée aux lecteurs. Mais cette interrogation résume en réalité parfaitement le programme éthique que Kramer poursuit depuis ses débuts. Car s'il est bien un sillon que l'auteure creuse dans ses romans, c'est celui de la singularité des parcours de vie qui exigent tout à la fois de patientes descriptions et des efforts de compréhension de la part des lecteurs. Ses plus récentes publications, Autopsie d'un père (2016), Une famille (2018) et Chronique d'un lieu en partage (20I6) explorent de nouvelles pistes - ou du moins, renouvellent sensiblement les voies génériques et techniques empruntées jusque-là, en s'emparant notamment de l'écriture ethnographique et en multipliant les points de vue. Cela contraste fortement avec la plupart de ses romans qui décrivent des groupes, que ce soit une famille (Autopsie d'un père, Les Vivants [2000], Fracas [2007]), un couple (Manu [1995], L'Adieu au Nord [2005], Onze ans plus tard [1999]), un groupe d'amis ou de collègues (Gloria), présentés à partir d'un point de vue unique. Ce dispositif de focalisation restreinte favorise l'immersion des lecteurs dans l'espace mental du personnage et leur permet de pénétrer de manière privilégiée dans des communautés très soudées, voire repliées sur elles-mêmes. À partir de ce point de vue singulier que les lecteurs sont invités à épouser, les tensions entre individu et collectif se dévoilent peu à peu. La voix narrative n'intervient pas : elle s'abstient de 
juger, évite les écueils du commentaire ou de l'interprétation, et refuse toute position de surplomb, laissant les lecteurs seuls arbitres des actions qui se déroulent.

Le monologue intérieur, largement réinvesti depuis les années 1980 par les romanciers français - pensons notamment à Laurent Mauvigner ou François Bon - fait entendre la parole de personnages déclassés ou marginaux; il joue ainsi volontiers sur le rythme syncopé des phrases et sur une recherche stylistique autour du dialogisme ${ }^{3}$. À l'inverse, l'usage que Kramer fait du point de vue se fonde sur une écriture blanche, presque impersonnelle. C'est par ce procédé que l'auteure, dont les capacités d'observation ont régulièrement été saluées par la critique, donne à voir l'univers dans lequel évoluent ses personnages. Afin d'assurer un grand réalisme dans les descriptions, le regard s'arrête volontiers sur les détails d'une mise, porte son attention sur des faits insignifiants du quotidien, scrute les gestes pour tenter de percer l'opacité des comportements d'autrui. Dans sa volonté de « sonder les détails infimes ou les non-dits» (Kramer, Delacrétaz \& Lepori 2007), la romancière immerge ses lecteurs dans des situations où s'installe un trouble, voire un malaise ou une angoisse.

Chaque roman propose ainsi une casuistique éthique à partir de drames à priori infimes et ordinaires. L'auteure interroge tour à tour les conséquences d'une trahison familiale, les ambiguïtés de l'amour maternel ou filial, les raisons et les effets de l'adultère ou les symptômes parfois ténus de la violence domestique. Bien que de portée individuelle, ces événements microsociaux soulèvent une succession de questions qui touchent la communauté dans son ensemble et évoquent, quoique par la bande, des problèmes de justice, de politique, de solidarité et, plus largement, de société. À partir de cette gamme de situations et de milieux variés, d'une petite bourgeoise de province à des environnements plus modestes, de personnages démunis ou sans voix, Kramer nous propose d'expérimenter la complexité des rapports humains et de comprendre que les responsabilités et les failles sont partagées. À y regarder de plus près, il est toujours difficile d'attribuer les torts à un seul personnage. Les plus coupables d'entre eux sont aussi, à une autre échelle, les victimes d'injustices, notamment de rapports de domination sociale, symbolique ou sexuelle.

L'exemple de L'Adieu au Nord (2005) nous convaincra du fait que Kramer travaille des questions éthiques au double niveau de la représentation et de l'expérience de lecture. Alors que ce récit semble tenir à quelques bribes intimes, il soulève des questions macrosociales importantes, mettant en scène, comme beaucoup de ses romans, la progressive implosion du commun, sa mise en crise et le " désastre à venir » (2005: 137). Construit en trois

3 À ce propos, on consultera les travaux de Frédéric Martin-Achard (2017). 
parties, comme une tragédie, L'Adieu au Nord relate la relation tumultueuse entre Patricia, une jeune fille de I7 ans et Alain, un ouvrier agricole précaire de 30 ans. Sous ses poses lascives et effrontées qui semblent tout autant destinées à éprouver son pouvoir de séduction sur les hommes qu'elle croise, qu'à provoquer son père supposément violent, Patricia élabore un plan pour fuir son quotidien sans horizon. Elle emmène Alain dans son sillage, lui qui, trop gauche pour séduire une femme, rêve de sortir de sa solitude et de connaître une véritable histoire d'amour. Leur départ en Angleterre, où Patricia s'est débrouillée pour obtenir un emploi de jeune fille au pair, révèle, par contraste, l'inadaptation d'Alain qui dépend financièrement et linguistiquement de sa compagne. La grossesse inattendue de Patricia et le viol de Luce, sa jeune amie restée en France, incitent les amants à retourner dans leur ville d'origine pour y installer leur ménage. Le rapport de force se modifie alors peu à peu : Alain gagne sa vie et prend l'ascendant sur $\mathrm{Pa}$ tricia dont il est jaloux et qu'il n'hésite pas à menacer et à maltraiter. La jeune femme, désormais résignée et passive, mais aussi complétement dépendante pécuniairement de son compagnon, semble consentir à vivre en recluse. Elle se mure dans le silence et se replie sur la relation à son enfant à naître, excluant par là le futur père.

Le couple, petit noyau de communauté, semblait promettre aux personnages la sortie d'une pesante solitude. Pour Alain, le « sentiment d'abandon [s'avère] presque intolérable » (II5), au point qu'il ressent un véritable « effroi de l'imaginer [Patricia] affranchie de lui » (I9I). Quant à Patricia elle éprouve le « besoin de se savoir amarrée à quelqu'un » (I67). Pourtant, d'épanouissant, le lien fragile qui les unit devient de plus en plus écrasant : Alain réalise que sa compagne est « désormais irrémédiablement liée à lui » (I55), qu'« elle [est] piégée, attachée à lui d'une façon qui le paniqu[e] lui en fait autant qu'elle » (I37). Plus que le bonheur, les projets ou les soucis, c'est la "souffrance [qui] se partage » (I63) alors. Et c'est bien d'une solitude en partage dont il faut parler pour décrire leur situation paradoxale.

Pour exprimer le malaise et l'inconfort croissants dans lesquels la relation plonge les personnages, Kramer use abondamment d'oxymores et d'antithèses - procédés qu'on retrouve d'ailleurs dans toute son œuvre. Ainsi Patricia fait-elle preuve d'un « détachement fébrile » (I7), tandis qu'Alain éprouve un "douloureux bonheur » (22) puis une " angoisse délicieuse » (3I). Le tiraillement des sentiments est par ailleurs renforcé par l'incompréhension qui caractérise les échanges. Une grande partie des événements est décrite à partir du point de vue d'Alain, à qui les mots pour exprimer son désarroi et ses émotions semblent manquer. Mais chez ce personnage, plus encore que le défaut des mots, c'est l'incapacité à concrétiser ses envies et ses désirs qui frappe le lecteur. Aussi, Alain parle « d'un bonheur dont 
l'imagination lui manqu[e] » (I59) et se trouve incapable d'agir et d'imaginer d'autres modes de vie.

De prime abord, l'intrigue de L'Adieu au Nord ne mobilise pas de manière évidente de grandes questions sociales, mais relate plutôt l'histoire des déboires amoureux d'un couple improbable. Pour autant, le récit ne laisse pas indifférent et nous invite à interroger de manière critique les enjeux de cette intrigue minimale. Une lecture manichéenne des actions des deux personnages s'avère impossible, tant ils sont à la fois, ou successivement victimes et bourreaux. Bourreaux de personnages plus faibles qu'eux, lorsqu'Alain frappe sa compagne enceinte ou quand Patricia manipule sa fragile amie Luce. Mais aussi victimes d'une précarité qui les laisse sans ressources, contraints à vivre dans des conditions matérielles difficiles et sans véritables attaches affectives. Cette vulnérabilité socio-économique les voue, en somme, à des réactions parfois violentes, aux éclats de colère. Leurs motivations et leurs actions, sans être excusables, s'éclairent alors d'un nouveau jour et leur condamnation paraît moins aisée. Kramer nous convie à juger, à évaluer ou à compatir, et non à condamner ses personnages : car pour l'auteure «si l'on se donne la peine de creuser dans la vie intérieure des gens, on s'aperçoit que tout peut être expliqué. Condamner est, de [s]on point de vue, pur manque d'imagination, incapacité à se mettre à la place de l'autre » (Kramer, Delacrétaz \& Lepori 2007). La littérature, en ce sens, serait plus encore qu'un exercice d'imagination, une invitation à s'impliquer en jetant un regard critique sur le monde afin de réactiver des solidarités perdues ou d'en créer de nouvelles.

\section{Solitude et imagination}

On l'a vu, la solitude ronge les personnages de L'Adieu au Nord (2005) et les rattrape au cour même du couple. Si Alain comme Patricia se résignent à rester ensemble malgré la peur et l'insatisfaction, c'est qu'ils s'avèrent tous deux incapables d'imaginer et de croire à un autre type de bonheur ou d'inventer une autre issue à leur relation. Alain imagine des scénarios virtuels qu'il ne parvient ni à réaliser ni à partager, tandis que sa compagne semble tout bonnement en être dépourvue : elle manque tout autant de capacités créatrices que d'un répertoire de situations semblables susceptibles de la guider dans ses choix.

Ces deux pans de l'imagination structurent en réalité toute l'œuvre de Kramer. Les personnages dont on suit le point de vue et les réflexions ne cessent de supposer, d'imaginer et d'inférer ce que pensent les autres à partir de leurs gestes ou de leurs paroles. Les verbes de supposition et les modalisateurs envahissent le texte et laissent poindre la difficulté que les personnages éprouvent à entrer en communication. Comme autant de chimères, les innombrables hypothèses n'indiquent finalement que le gouffre d'in- 
compréhension qui séparent les personnages : " ils se taisaient, chacun imaginant pour soi, dans le secret indicible de son imagination, l'enfant errant "(Kramer I995 : 196). Les personnages de Manu (I995) craignent la violence de la réalité ou de ses conséquences, lorsqu'ils imaginent tous deux le sort possible du très jeune enfant qu'ils ont laissé seul sur une route dangereuse. Ils se taisent pour conjurer les visions fantasmatiques qui peuplent leur esprit et dont ils pensent qu'elles pourraient se concrétiser s'ils les laissaient affleurer. D'autres se préservent en se refusant tout simplement à imaginer : par égoïsme, ignorance ou réflexe d'autoconservation, ils repoussent la souffrance qui les entoure et résistent à la compassion, comme à toute forme d'émotion. Dans Gloria (2013), le personnage éponyme se montre « imperméable au malheur abstrait des autres » (43). Michel, l'ancien assistant social qu'elle sollicite pour l'aider à obtenir un nouveau logement s'étonne de la voir si exigeante et sûre d'elle-même, au mépris du contexte dans lequel elle évolue. Observant Gloria, il en vient à la conclusion que « [c]e n'était finalement rien d'autre que cela sa différence : cette totale incapacité à être ailleurs qu'en elle-même » (43). Mais l'empathie et l'imagination de Michel, qui ne lui avaient jamais fait défaut jusque-là, semblent le quitter, au point que « lui qui avait côtoyé tant de ces vies en marges pendant des années ne se sentait plus ni la tolérance ni surtout la folle abnégation qu'il fallait pour en accepter tout sans juger. Je suis devenu égoïste ou beaucoup trop réaliste, songea-t-il »(IO2). Les termes employés par le personnage sont particulièrement importants, tant ils renvoient à une éthique, à des valeurs, en somme, à une conduite qu'il n'est plus à même de suivre. "Tolérance ", " abnégation », « accepter tout sans juger ", voilà ce dont Michel n'est plus capable. Ce désenchantement et cette incompréhension face aux actes, aux humiliations et aux souffrances d'autrui nous signalent les difficultés que représente la compassion. Plus encore, l'exemple nous permet de mesurer les dangers du retrait et les conséquences de l'indifférence : une communauté définitivement fracturée et inégale.

Dans Les Vivants (2000), Benoît, l'oncle de deux enfants qui meurent accidentellement sous sa surveillance, est d'abord saisi d'effroi par le drame, et partage la douleur de sa sœur dévastée. Mais, assez rapidement, le sentiment de pitié disparaît et Benoit, comme la plupart de son entourage, «appr[end], aux dépens de Louise [sa sœur], à s'efforcer de ne surtout pas penser » (44) et « préf[ère] cela : ne surtout rien voir ni savoir de leur malheur » (45). Le manque de compassion, le rejet de la douleur d'autrui condamne le personnage de Louise à un total isolement : son compagnon et son frère l'abandonnent, tandis que, chez sa mère, « la lassitude déjà prenait le pas sur la pitié - elle était trop vivante pour compatir au-delà du supportable » (I53). Ici aussi, le refus d'imaginer et de compatir a pour corollaire la dislocation de la communauté. 
L'idée que le défaut d'imagination condamne à la solitude et entrave toute forme de solidarité est récurrente chez Kramer. Quel que soit le milieu décrit, les thèmes abordés ou les questionnements des personnages, le rapport à l'imagination dessine, dans cette œuvre, les partages possibles au double sens évoqué par Jacques Rancière, à savoir un "commun partagé " et des "parts exclusives » (Rancière 2000 : I2). Les plus récents textes de l'auteure, Autopsie d'un père (2016), Une famille (2018) et, dans un genre tout autre, Chronique d'un lieu en partage (2016), mettent justement davantage l'accent sur ces partages en réfléchissant non seulement à la pluralité des « nostrations ", c'est-à-dire aux appartenances diverses et parfois simultanées qu'un même individu peut revendiquer, mais aussi aux effets complexes qu'elles engendrent. C'est ici que la romancière rejoint les préoccupations d'intellectuels contemporains qui, comme Jean-Christophe Bailly, Tristan Garcia, Marielle Macé - ou Rorty avant eux -, interrogent l'usage du pronom « nous ». Alors qu'il peut se parer de connotations politiques et sociologiques positives lorsqu'il se pense comme une « jonction » (Benveniste I966: 233), ce pronom charrie également avec lui les échos de discours d'appartenance et d'identité fondés sur des logiques d'opposition, et c'est cela que Kramer sonde dans ces derniers textes.

\section{«Nous»}

De cette attention particulière à la formation de communautés tantôt ouvertes, tantôt exclusives, Autopsie d'un père (2016) est sans doute l'exemple le plus remarquable. Le roman raconte la relation compliquée, voire conflictuelle, qu'Ania entretient avec son père, Gabriel, un journaliste dont les convictions politiques ont progressivement passé d'une gauche plutôt libérale à un discours proche de la droite identitaire. À l'annonce du suicide de son père, Ania redécouvre la vie du défunt dont elle s'était éloignée, et se met à côtoyer son entourage, le temps des préparations des obsèques. Elle réalise alors que son père a pris publiquement la défense des agresseurs d'un immigré comorien, mort sous leurs coups, et que nombreux sont ceux qui, dans le cercle de son père, voient dans leur geste la sauvegarde d'une identité nationale en péril. Au travers de cette histoire familiale, transparaît celle de mondes sociaux qui s'affrontent et de collectifs qui se heurtent. C'est une entrée particulière dans notre présent tumultueux et une manière de réfléchir aux liens qui nous rassemblent.

Tout distingue Ania de son père : elle vit dans une banlieue défavorisée et vient de se séparer de Novak, un immigré serbe avec qui elle a eu un enfant malentendant. Gabriel, lui, réside entre Paris et la campagne d'Ile de France, avec Clara, sa jeune épouse, qu'Ania ne connaît pas. Ils évoluent ainsi dans des sphères culturelles opposées. Faisant l'expérience d'une forme de mépris social, Ania conçoit son union avec Novak comme une fa- 
çon de se « veng[er] du monde offensant de son père » (28) et s'enorgueillit du fait que son fils, maintenu depuis sa plus tendre enfance à l'écart de cet univers, n'ait « aucune expérience ni même aucun soupçon de ces milieux intouchables et cultivés où elle s'était sentie tellement déficiente étant gamine » (24). Mais, si Ania se sent appartenir à un autre monde et s'est appliquée à échapper, coûte que coûte, à ces milieux hermétiques qui lui sont désormais complétement étrangers, elle ne se reconnaît pas pour autant dans une communauté de personnes précaires qu'elle rencontre dans les rues de sa cité. En réalité, à la vue d'un homme prêt à s'emparer d'objets laissés sur un tas d'ordures, Ania éprouve certes de l'inconfort, mais elle doit surtout s'avouer que « la préoccupation de ces vies si précaires, échouées là de plus en plus nombreuses, l'accabl[e] d'une angoisse et de réactions de rejet qu'elle n'arriv[e] pas toujours à raisonner » (IO4). Le personnage d'Ania semble en définitive se situer en marge des communautés constituées : si elle se tient à distance de celle de son père, elle repousse également celle des plus démunis de son quartier et semble finalement se replier sur le noyau de la famille nucléaire qu'elle forme avec son fils. En filigrane, le récit évoque donc toute une série d'antagonismes qui structurent, ou fracturent, la société. Affleure en effet une opposition entre la campagne, la ville et la banlieue ; entre les personnes souffrant de handicap et celles qu'on dit valides ; entre les immigrés et les locaux, entre les privilégiés et ceux qui se sentent oubliés, entre les jeunes et les vieux.

Ces cercles multiples croisent d'autres constellations, des collectifs qui s'expriment plus strictement au moyen du pronom «nous ». Les personnages eux-mêmes s'en saisissent pour se définir et marquer leur appartenance à une ou plusieurs communautés. En retournant dans le village où elle a grandi, Ania réalise à quel point les « nous » se configurent et se modifient au gré des discours : ainsi, elle est surprise lorsque le gardien de la maison de Gabriel, parlant de l'enterrement à venir, se sert du pronom « nous » pour dessiner une communauté familiale dont elle est paradoxalement exclue : «Ce nous les englobait, eux [le couple de gardiens] et Clara, dans un lien nouveau de filiation. [...] Clara leur apportait plus de satisfaction et de gages d'amitié que la gamine secrète et menteuse qui avait désespéré toutes leurs attentes » (II9). Ici, le « nous » sert à recomposer une famille qui, bien que soudée par l'affliction, n'a pas de véritable lien de parenté ; il autorise surtout l'exclusion de ceux qui ne correspondent pas au comportement valorisé par cette lignée imaginée. Ce type de communauté affective, qui recourt au lexique de la famille pour s'exprimer, se déploie dans ce roman dès le titre. Ania, qui veut, selon l'expression consacrée, «tuer le père ", éprouve en effet, et bien malgré elle, " comme une parenté » avec Clara dont elle perçoit, à son allure, l'éducation bourgeoise. Cela réveille d'ailleurs, chez elle, et non sans trouble, « un sentiment d'appartenance de classe qu'elle ne se connaissait pas » (I29). 
Le «nous » apparaît de manière particulièrement brutale lorsqu'il permet de fédérer des personnages autour de positions idéologiques, voire politiques. En soutenant les deux jeunes assassins de l'immigré comorien, Gabriel a attisé les rancœurs d'une partie des habitants de cette campagne délaissée qui craint que « [leur] civilisation [...] crève» (99), et qui voit dans la défense d'une identité nationale un salut à sa situation. Dans le même temps, il a ravivé la colère de ceux qui, à l'inverse, prônent davantage de solidarité envers les plus démunis. Dans le roman, les deux camps s'affrontent régulièrement et avec véhémence. En témoigne l'hostilité marquée des opposants à Gabriel qui cherchent notamment à perturber la cérémonie funèbre. Alors qu'Ania s'étonne de cette violence, sa belle-mère lui rétorque " avec un mépris formidable » que «ce sont des idéalistes », et que "ça fait un moment qu'ils nous emmerdent » (159). Le clivage politique et idéologique entre les «idéalistes » et ce "nous » aux contours flous, qui rassemble les proches de Gabriel et les partisans de la mouvance identitaire, se termine dans un violent incendie qui ravage la maison où vit un couple " d'idéalistes ». Cette déflagration métaphorise, plus largement, celle du commun. La possibilité même de maintenir ce «nous humains » semble illusoire, et l'autopsie à laquelle procède le roman pourrait bien être celle de la société contemporaine, tant il paraît difficile de la fédérer à nouveau dans un lien de solidarité réel. Il revient donc aux lecteurs d'éprouver la place de chacun des personnages et, une fois le livre clos, d'imaginer des manières de refaire communauté.

Dans Chronique d'un lieu en partage (2016), l'auteure, qui s'empare cette fois d'une forme proprement documentaire ou ethnographique, prolonge sa réflexion sur la communauté. À l'inverse du constat pessimiste que dresse Autopsie d'un père, ce dernier ouvrage semble chercher à réparer la dissension qu'esquissait le texte fictionnel, en montrant comment ce qu'elle nomme une « utopie sociale » peut fonctionner dans la réalité. Kramer y présente en effet un montage de témoignages de personnes vivant en communauté dans un ancien Carmel. L'intérêt du lieu réside dans sa capacité à « mélanger les milieux sociaux ", puisque s'y croisent des personnes précaires au parcours sinueux, des touristes de passage et une équipe encadrante. Pour Kramer, qui explore les liens entre individu et collectif depuis le début de son œuvre, la diversité sociale est une condition nécessaire pour que l'on puisse " arriver à une société plus juste »(Kramer \& Garbely $2017: 2$ ). La solidarité, qui semble sinon inatteignable, du moins fragile dans ses textes de fiction, paraît trouver ici une actualisation possible. Plus encore, elle rejoint l'idéal de Rorty que je citais en introduction, puisqu'il s'agit, pour l'un comme pour l'autre, de penser les fondements d'une utopie qui permettrait de restaurer la solidarité humaine et d'« englober dans le champ du "nous" des gens très différents de nous » (1993:263). 
Les « histoires singulières rendent le monde intelligible ». Pour une politique de la littérature

Nouer le singulier et le collectif, comprendre ce que nous avons en commun à partir de parcours et de points de vue singuliers, et par cela, rendre "le monde intelligible » (Kramer \& Garbely 2017 : 2). Voilà le programme éthique que se fixe Kramer depuis ses débuts. Son œuvre dessine une mosaïque de portraits qui questionne notre présent et nos manières de vivre ensemble. Sans jamais imprimer de morale ou de thèse univoque à ses romans, l'auteure nous invite à éprouver les modes d'existence de ces autres que nous ne voyons plus, à les considérer avec empathie. Cette circulation dans des mondes sociaux variés fonctionne alors comme une incitation à recréer des liens, par-delà la fiction, à laquelle Kramer confère une véritable puissance. Car c'est par le truchement d'histoires et de récits immersifs qu'elle cherche à impliquer les lecteurs en suscitant, chez eux, le désir d'imaginer des alternatives au monde parfois désenchanté qu'elle représente. En cela, on peut dire que la pratique de la littérature, chez Kramer, est triplement politique : elle donne la parole à des personnages marginaux ou défavorisés ; elle appelle une lecture critique de phénomènes intimes et collectifs et espère, finalement, fédérer des solidarités et des communautés pour inventer de nouveaux liens.

\section{Bibliographie}

Bailly, Jean-Christophe, «"nous" ne nous entoure pas », Vacarme, vol. 69, n4, 20I4, pp. I72-I95.

Benveniste, Émile «Structure des relations de personne dans le verbe », Problèmes de linguistique générale, I, Paris, Gallimard, I966, pp. 225-236.

Blanckeman, Bruno, «De l'écrivain engagé à l'écrivain impliqué. Figures de la responsabilité littéraire au tournant du XXI e siècle ", Catherine Brun \& Alain Schaffner (dir.), Des écritures engagées aux écritures impliquées. Littérature française ( $x x^{e} / \mathrm{XxI}^{e}$ siècles), Dijon, Éditions universitaires de Dijon 2015, pp. I6I-I69.

Booth, Wayne C., The Company We Keep. An Ethics of Fiction, Berkeley, University of California Press, 1988.

Brun, Catherine \& Schaffner, Alain (dir.), Des écritures engagées aux écritures impliquées. Littérature française ( $x x^{e} / x_{x I} I^{e}$ siècles), Dijon, Éditions universitaires de Dijon, 2015.

Diamond, Cora, L'Importance dêtre humain. Et autres essais de philosophie morale, trad. par Emmanuel Halais, Paris, PUF, 20II.

Garcia, Tristan, Nous, Paris, Grasset, 2016. 
Kieffer, Morgane, «La possibilité du monde : fictions critiques et réalisme adressé dans le contemporain français ", RELIEF - Revue électronique de littérature française, vol. I3, $\mathrm{n}^{\circ} \mathrm{I}$, juillet 2019, pp. 13-27.

Kramer, Pascale, Manu, Paris, Calmann-Lévy, 1995.

-. Onze ans plus tard, Paris, Calmann-Lévy, 1999.

-. Les Vivants, Paris, Calmann-Lévy, 2000.

-. Retour d'Uruguay, Paris, Mercure de France, 2003.

-. L'Adieu au Nord, Paris, Mercure de France, 2005.

-. Fracas, Paris, Mercure de France, 2007.

-. Gloria, Paris, Flammarion, 2013.

-. Chronique d'un lieu en partage, Ivry-sur-Seine, Les Éditions de l'Atelier/ Éditions Ouvrières, 2016.

-.Autopsie d'un père, Paris, Flammarion, 2016.

-. Une famille, Paris, Flammarion, 2018.

Kramer, Pascale, Delacrétaz, Aline \& Lepori, Pierre, "Pascale Kramer », Viceversa Littérature, 2007, en ligne : http://www.culturactif.ch/viceversa/ kramer.htm (consulté le Io/oI/2020).

Kramer, Pascale \& Garbely, Nathalie, «Entretien avec Pascale Kramer », 20I7, en ligne : https://romandesromands.ch/files/2017/o2/Entretien-avec-Pascale-Kramer.pdf (consulté le Io/or/2020).

Macé, Marielle, " "Nouons-nous". Autour d'un pronom politique », Critique, vol. 84I-842, n6, 20I7, pp. 469-483.

Martin-Achard, Frédéric, Voix intimes, voix sociales. Usages du monologue romanesque aujourd'hui, Paris, Classiques Garnier, 2017.

Message, Vincent, Romanciers pluralistes, Paris, Seuil, 2013.

Nussbaum, Martha, L'Art d'être juste. L'imagination littéraire et la vie publique, traduit par Solange Chavel, Paris, Climats, 2015 [1997].

Rorty, Richard, Contingence, ironie \& solidarité, traduit par Pierre-Emmanuel Dauzat, Paris, Armand Colin, 1993 [1989].

Viart, Dominique, «Le moment critique de la littérature. Comment penser la littérature contemporaine? », Bruno Blanckeman et Jean-Christophe Millois (dir.), Le Roman français aujourd'hui : transformations, perceptions, mythologies, Paris, Prétexte, 2004, pp. II-35. 
